\title{
A Novel Vector Network Analyzer
}

\author{
Karel Hoffmann, Member, IEEE, and Zbynek Škvor, Senior Member, IEEE
}

\begin{abstract}
A calibration procedure for a perturbation two-port vector network analyzer is presented. It consists of a variable perturbation two-port placed between a device-under-test and a scalar network analyzer. A measured vector reflection coefficient is determined on the basis of amplitude-only measurements. A full correction of systematic errors is possible. The new principle was experimentally confirmed in the frequency band up to $14 \mathrm{GHz}$. The results enable prediction of an add-on for scalar analyzers, enabling for vector measurements.
\end{abstract}

Index Terms-Calibration, measurement, measurement errors, measurement standards.

\section{INTRODUCTION}

$\mathbf{V}$ ECTOR NETWORK analyzers (VNA's) based on frequency conversion are widely used for wide-band vector measurements. They enable in principle the elimination of all systematic errors if a proper calibration/correction procedure is applied. A lot of sophisticated methods have been developed, see, for example, [1]. The greatest disadvantage of this systems is its complexity which results in high cost. Six-port VNA's, see [2]-[4], offer cheaper solutions. They also enable full correction of systematic errors.

On the other hand, scalar network analyzers (SNA's) are simpler and cheaper, but they provide only scalar information. Moreover, in principle, they cannot be fully calibrated to remove systematic errors, so that they provide only an approximate value of a measured amplitude, even if precise directional bridges and detectors are used.

A new concept for vector measurements based on a SNA and perturbation two-port (PTP) was suggested in [5] and [9]. The purpose of this paper is to present a new calibration procedure and first experimental results with this VNA based on SNA and PTP. The calibration procedure enables full correction of systematic errors for both vector and scalar measurements.

\section{THEORY}

Fig. 1 shows the basic measurement arrangement of the PTP vector network analyzer. Reflection coefficient $\rho_{\text {DUT }}$ of the device under test (DUT) in the reference plane B, which ought to be determined, is transformed over the PTP to the reference plane A. Its amplitude $\left|\rho_{\mathrm{M}}\right|$ is measured by an ideal scalar network analyzer. If $S$-parameters $S_{i j}$ of the PTP are known,

Manuscript received March 30, 1998; revised September 3, 1998. This work was supported by the Grant Agency of Czech Republic under Grant $102 / 97 / 1224$.

The authors are with the Faculty of Electrical Engineering, Czech Technical University, 16627 Prague 6, Czech Republic.

Publisher Item Identifier S 0018-9480(98)09234-5.

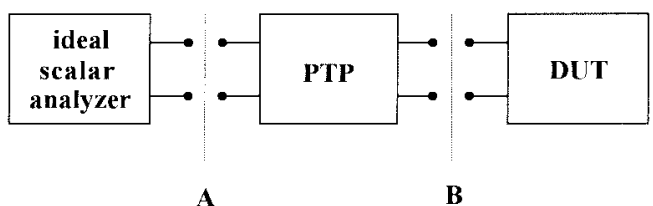

Fig. 1. Measurement arrangement of the PTP vector network analyzer.

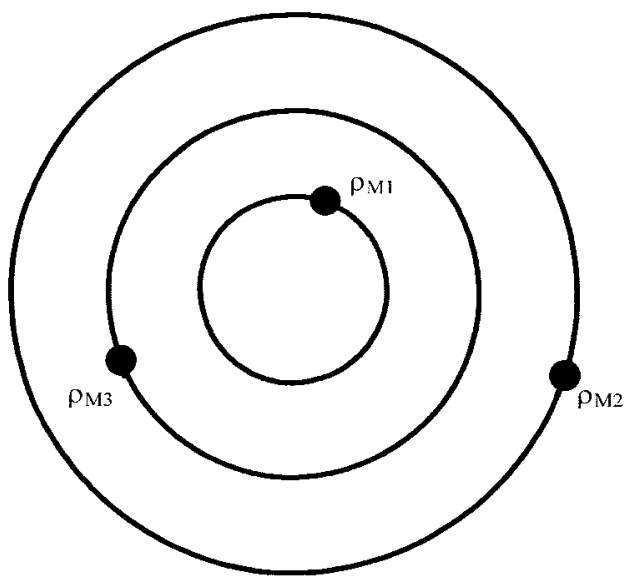

Fig. 2. Circles in the complex plane $\rho_{\mathrm{A}}$.

the relation between $\rho_{\text {DUT }}$ and $\rho_{\mathrm{M}}$ is given by

$$
\rho_{\mathrm{M}}=S_{11}+\frac{S_{12} S_{21} \rho_{\mathrm{DUT}}}{1-S_{22} \rho_{\mathrm{DUT}}} .
$$

Equation (1) forms a conformal transformation between complex planes $\rho_{\mathrm{A}}$ and $\rho_{\mathrm{B}}$ of the reflection coefficients in the reference planes $\mathrm{A}$ and $\mathrm{B}$. Therefore, circles in the complex plane $\rho_{\mathrm{A}}$ with the radius $\left|\rho_{\mathrm{M}}\right|$ are transformed by (1) into circles in the complex plane $\rho_{\mathrm{B}}$ and vice versa, see Figs. 2 and 3 .

Circles in the complex plane $\rho_{\mathrm{A}}$ can be determined by the measurement of the corresponding modules of the reflection coefficients $\left|\rho_{\mathrm{M}}\right|$ in the reference plane A for different settings of the PTP $S$-parameters. The common intersection of the corresponding circles in the complex plane $\rho_{\mathrm{B}}$ determines the desired reflection coefficient $\rho_{\text {DUT }}$ of the DUT in the reference plane B. At least three circles are necessary for the unique determination of $\rho_{\text {DUT }}$. This principle is similar to the theory of the six-port VNA's. Contrary to constant sixport $S$-parameters in the new concept, the PTP parameters are different for the individual settings. Therefore, the six-port theory cannot be used. $\rho_{\text {DUT }}$ can be determined only if $S$ parameters of the PTP are known. They must be determined in the process of calibration. 


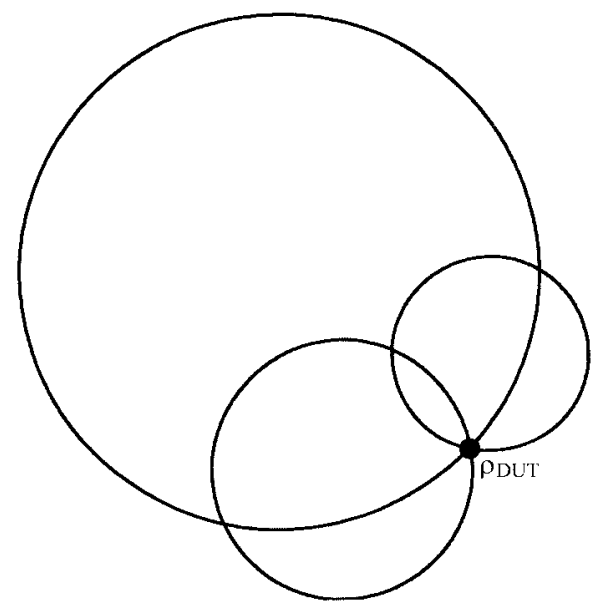

Fig. 3. Corresponding circles in the complex plane $\rho_{\mathrm{B}}$.

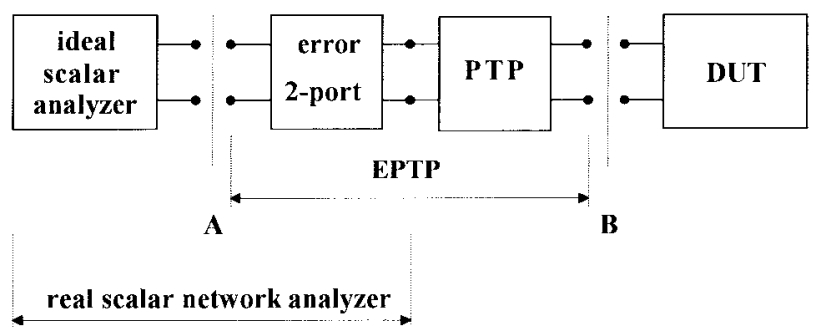

Fig. 4. Description of scalar network analyzer systematic errors.

Fig. 1 shows an ideal scalar analyzer with zero measurement errors considered. However, it is a well-known fact that a real scalar analyzer always has some systematic errors that cannot be removed by any scalar only calibration and correction. It will be shown that in the new concept of the PTP VNA there is a possibility to overcome this fundamental obstacle.

\section{CALiBRAtion}

The standard process commonly used for VNA systematic error correction can be applied also on a real scalar analyzer. The error two-port and the PTP can be merged into one error perturbation two-port (EPTP), see Fig. 4, and its parameters for individual PTP settings must be determined in the calibration of the PTP VNA. A correct calibration of the EPTP is a cornerstone of the new concept.

Only amplitude scalar measurements of $\left|\rho_{M}\right|$ corresponding to the full known complex reflection coefficient $\rho_{\mathrm{C}}$ of properly chosen calibration standards can be used to determine unknown $S_{i j}$ parameters of the EPTP. Equation (1) yields

$$
\left|\rho_{\mathrm{M}}\right|=\left|S_{11}+\frac{S_{12} S_{21} \rho_{\mathrm{C}}}{1-S_{22} \rho_{\mathrm{C}}}\right|
$$

which can be used to form a proper system of equations. These equations are nonlinear, and a numerical method must be used to solve them.
The EPTP is a reciprocal two-port. Therefore there are only three complex or six scalar unknown parameters in (2). Unfortunately there are only five independent equations in the system based on (2) and therefore only five scalar unknowns can be determined. One remaining unknown parameter cannot be determined, and this is why the correct calibration of a scalar network analyzer alone is impossible. The physical interpretation of this fact can be given in the following way. One can imagine that the EPTP ends in the reference plane A by a line with the characteristic impedance equal to that of the SNA. The length of this line cannot be determined by any means by the only amplitude measurement in plane A. The length of the line determines the position of the $\rho_{\mathrm{M}}$ on corresponding circle in plane A, see Fig. 2.

In the new concept the sixth parameter does not need to be known for the correct determination of the complex $\rho_{\mathrm{DUT}}$. The missing information is added by at least three measurements of $\left|\rho_{\mathrm{M}}\right|$ for at least three different settings of the PTP. The sixth parameter can be defined arbitrarily and no matter how it is defined the correct $\rho_{\text {DUT }}$ is given by the common intersection of the corresponding circles, see Fig. 3. The only effect of the sixth parameter is that circles at Fig. 3 are turned around their centers, keeping the common intersection intact.

The calibration/correction procedure of the PTP was modeled and experimentally verified in the frequency band 2-2.5 GHz. A simple PTP in the coplanar waveguide structure was realized for this purpose. Five calibration standards: open, short, offset open, offset short, and match were used for the simulation and the experiment.

In the first step, the reflection coefficient of the calibration standards were transformed from plane B to plane A, see Fig. 1, with PTP measured $S$-parameters applied to obtain corresponding simulated "measured" amplitudes. These "measured" amplitudes were used in the second step to calculate simulated $S$-parameters of the PTP. The numerical algorithm solving the system of five nonlinear equations based on (2) was designed so that the original reflection coefficients of the calibration standards were obtained. It was discovered that the procedure yielded two different sets of the PTP $S$ parameters. Both solutions were thoroughly tested by the above-mentioned calibration/correction procedure in the whole region of $\rho_{\text {DUT }}<1$ with the result that they give the same and correct $\rho_{\text {DUT }}$ only if it equals the value of the calibration standard. For the other possible reflection coefficients of the measured load, they give two different $\rho_{\mathrm{DUT}}$ 's, one correct and the other a bit different and incorrect. It can be seen on Fig. 5 which displays the amplitude difference between correct and incorrect $\rho_{\mathrm{DUT}}$.

The problem was theoretically studied and the existence of the two and only two solutions was explained in the following way.

Equation (2) can be rearranged to the form, shown in (3) at the bottom of this page. It can be seen that the system of

$$
\left|\rho_{\mathrm{M}}=\right| S_{11}+\frac{\left|S_{12} \cdot S_{21}\right| \cdot\left|\rho_{\mathrm{C}}\right| \cdot \mathrm{e}^{j \cdot\left[\arg \left(S_{12} \cdot S_{21}\right)+\arg \left(\rho_{\mathrm{C}}\right)\right]}}{1-\left|S_{22}\right|^{2} \cdot\left|\rho_{\mathrm{C}}\right|^{2}-2 \cdot\left|S_{22}\right|\left|\rho_{\mathrm{C}}\right| \cdot \cos \left[ \pm\left|\arg \left(S_{22}\right)\right|+\arg \left(\rho_{\mathrm{C}}\right)\right]} \cdot\left[\mathrm{e}^{j \cdot \arg \left(\rho_{\mathrm{C}}\right)}-\left|\rho_{\mathrm{C}}\right| \cdot\left|S_{22}\right| \mathrm{e}^{ \pm j \cdot\left|\arg \left(\mathrm{S}_{22}\right)\right|}\right] \mid
$$




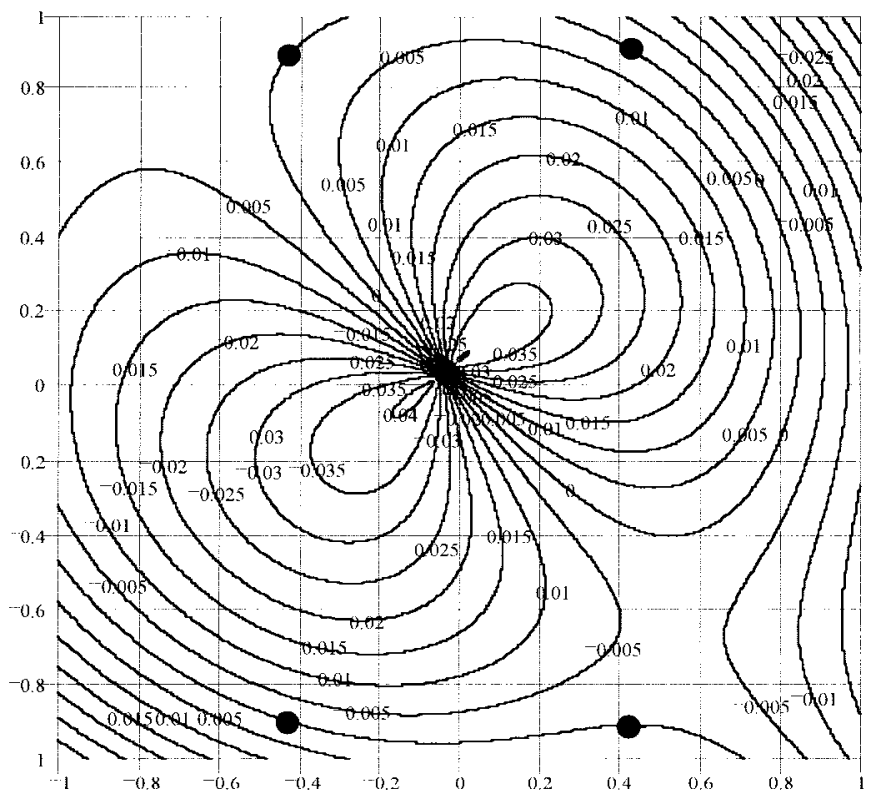

Fig. 5. The amplitude difference between the correct and the incorrect $\rho_{\text {DUT }}$. Four points correspond to reflective calibration standards.

equations based on (3) gives for only five calibration standards two solutions in accordance with the sign of the $\arg \left(S_{22}\right)$ parameter.

The unique and correct solution for the $\rho_{\text {DUT }}$ can be achieved only if one at least partially known calibration standard is added to the calibration set to eliminate the wrong sign in (3). Therefore, five and a "half" fully known different calibration standards is a theoretical minimum demand on the calibration set for the PTP VNA calibration. One or two additional calibration standards can be recommended to obtain a wider frequency band and greater robustness of the numerical solution.

\section{EXPERIMENTAL RESULTS}

To verify correctness of the whole calibration and correction algorithm, a simple voltage-controlled PTP in the structure of CPW was designed and realized. Match, short, and five offset shorts were used for calibration to achieve a wider frequency band. The offset shorts were realized in the structure of the air $7 \mathrm{~mm}$ coaxial line with APC7 connectors. A 3-dB attenuator with APC7 connectors terminated with short was used to test the measurement ability of the PTP VNA. The reflection coefficient of this load ( $3 \mathrm{~dB}$ attenuator plus short) was measured on the VNA (a computer-controlled HP 8410 VNA with a correction method applied) and on the PTP VNA in the frequency band 8-14 GHz. HP 8757 E SNA was used to create the PTP VNA. A numerical algorithm similar to [6] was used to calculate $\rho_{\text {DUT }}$.

Fig. 6 shows a comparison between phases of the test DUT obtained by the new method and the phases measured by the "classic" VNA. As can be seen, good general agreement of both traces on the majority of the frequency band has been achieved. This general agreement confirms correctness of the calibration/correction procedure. As far as the authors know, this is the first published result of a phase measurement

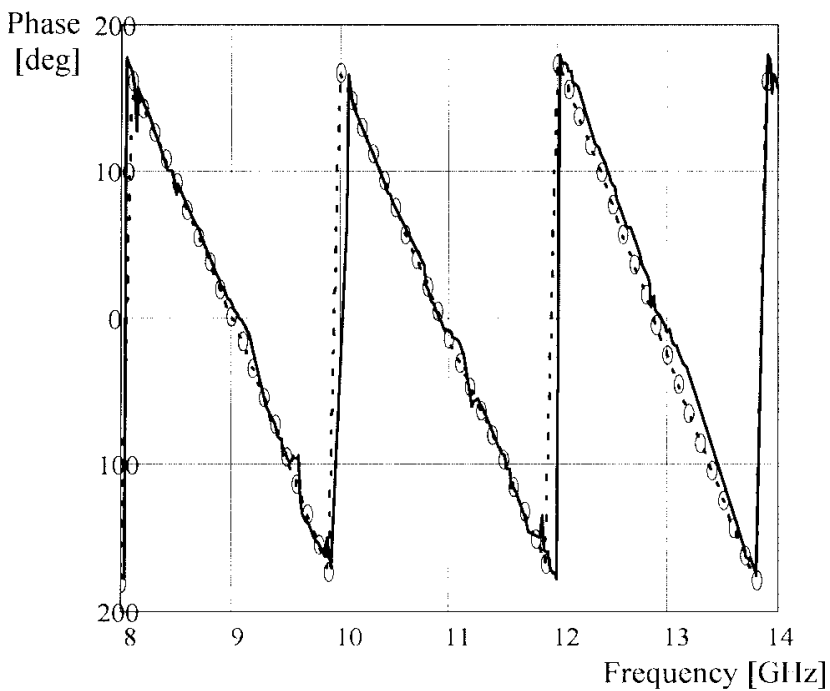

Fig. 6. The phase of the reflections coefficient measured on VNA (o-o-o) and PTP VNA (-). No averaging nor data smoothing used

obtained by this procedure. The differences of both traces are typically in the order of several degrees and correspond more or less to errors of the first experimental results obtained with the six-port VNA [7], [8]. The noise in the results presented is believed to be caused mainly by the fact that the process was not automated, e.g., collecting all the data for calibration and measurement at 401 frequency points took about six hours. Therefore, a lot of time has been left for equipment instability. Manually controlled PTP settings also did not assure top reproducibility.

\section{CONCLUSION}

The calibration/correction procedure for the PTP VNA was developed and experimentally verified. The new vector network analyzer system based on scalar measurement only was realized as an addition to a commercially available SNA. The viability of the new system was experimentally verified and confirmed.

The PTP VNA with the calibration/correction procedure developed opens the possibility to develop VNA's as an extension of current scalar network analyzers not only in microwave band, but also in millimeter and submillimeter frequency bands. Moreover, if such an improved scalar network analyzer is used for scalar measurements only, the full correction of its systematic errors is possible.

The simplicity of the PTP VNA promises low cost of the whole system. The authors hope to integrate the whole calibration and measurement calculations as well as data acquisition into one program soon, reducing measurement time and error. A simple add-on, controlled PTP is expected to provide vector reflection measurement to scalar analyzer users.

\section{REFERENCES}

[1] K. Silvonen, "LMR 16-A self-calibration procedure for a leaky network analyzer," IEEE Trans. Microwave Theory Tech., vol. 45, pp. 1041-1049, July 1997. 
[2] G. F. Engen, "The six-port reflectometer: An alternative network analyzer," IEEE Trans. Microwave Theory Tech., vol. MTT-25, pp. 1075-1083, Dec. 1977.

[3] C. A. Hoer, "A network analyze incorporating two six-port reflectometers," IEEE Trans. Microwave Theory Tech., vol. MTT-25, pp. 1070-1074, Dec. 1977.

[4] V. Bilik and J. Bezek, "Thin film lumped six-port vector reflectometer for 10-1900 MHz," in Conf. Proc., MITEKO 1995, University of Pardubice, Czech Republic, June 22-23 1995, pp. 181-184.

[5] Z. Škvor and K. Hoffmann, "Vector measurement with scalar analyzer," in MIOP'95 Conf. Proc., Sindelfingen, Germany, May 30-June 1, 1995, pp. 30-33.

[6] W. Wiatr and M. Schmidt-Szalowski, "Noise and small-signal characterization of GaAs FET's in multistate radiometer system," in Proc. Int. IEEE Workshop on Experimentally Based FET Device Modeling \& Related Nonlinear Circuit Design, University of Kassel, Germany, July 17-18, 1997, pp. 35.1-35.8

[7] H. M. Cronson and L. Susman, "A six-port automatic network analyzer," IEEE Trans. Microwave Theory Tech., vol. MTT-25, pp. 1086-1091, Dec. 1977.

[8] C. A. Hoer and K. C. Roe, "Using an arbitrary six-port junction to measure complex voltage ratios," IEEE Trans. Microwave Theory Tech., vol. MTT-23, pp. 978-984, Dec. 1975.

[9] K. Hoffmann and Z. Škvor, "A novel vector network analyzer," in 1998 IEEE MTT-S Dig., Baltimore, MD, June 7-12, 1998, pp. 953-956.

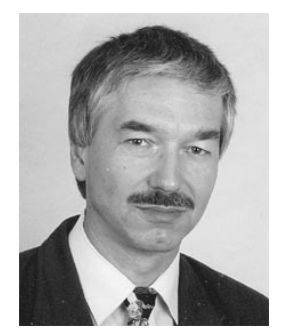

Karel Hoffmann (M'93) was born in Prague, Czech Republic. He graduated from the Czech Technical University, Faculty of Electrical Engineering, in 1974 (Hons). His diploma thesis dealt with a Gunn microwave oscillator.

$\mathrm{He}$ is with the Department of Electromagnetic Field of Czech Technical University in Prague. In 1990 and 1991, he spent four months at the University Tor Vergata, Rome. He carried out research on precise microwave measurement correction methods. The results formed the kernel of his habilitation thesis. Since 1993, he has been an Associate Professor at the Czech Technical University, Faculty of Electrical Engineering. His professional activities are focused on active and passive microwave integrated circuits, correction methods for precise microwave measurement, development of new microwave vector network analyzers, and modeling of microwave packages.

Currently, Mr. Hoffmann is a Chair of the MTT/AP/ED joint chapter of the Czechoslovakia section of IEEE. He is one of the cofounders of the "International Traveling Summer School on Microwaves and Lightwaves."

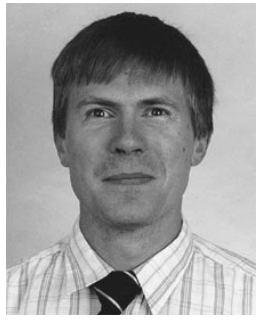

Societies Chapter.
Zbynek Škvor (M'91-SM'96) received the M.Sc. and $\mathrm{Ph} . \mathrm{D}$. degrees in radioelectronics from the Czech Technical University in Prague.

$\mathrm{He}$ is a Deputy Head of the Department of Electromagnetic Field, Czech Technical University. His research activities include microwave measurements, electromagnetic field modeling, and CAD. He has authored/coauthored more than 100 papers and reports.

He serves as a Chairman of the Czechoslovakia Section IEEE and a past Chairman of its MTT/AP 